\title{
Association of Health Insurance Literacy with Health Care Utilization: a Systematic Review
}

\author{
Brian F. Yagi, MD, JD' , Jamie E. Luster, MPH², Aaron M. Scherer, $P h D^{3}$, \\ Madeline R. Farron, MPH, MA ${ }^{2}$, Judith E. Smith, MSLIS4, and Renuka Tipirneni, \\ $M D, M S C^{2,5}$
}

\begin{abstract}
'Department of Internal Medicine, University of Michigan, Ann Arbor, MI, USA; ${ }^{2}$ Department of Internal Medicine, Division of General Medicine, University of Michigan, 2800 Plymouth Road, Bldg 16, Rm 419W, Ann Arbor, MI, USA; ${ }^{3}$ Department of Internal Medicine, Division of General Internal Medicine, University of lowa, lowa City, IA, USA; ${ }^{4}$ Taubman Health Sciences Library, University of Michigan, Ann Arbor, MI, USA; ${ }^{5}$ Institute for Healthcare Policy and Innovation, University of Michigan, Ann Arbor, MI, USA.
\end{abstract}

BACKGROUND: Given increasing numbers of people experiencing transitions in health insurance due to declines in employer-sponsored insurance and changes in health policy, the understanding and application of health insurance terms and concepts (health insurance literacy) may be important for navigating use of health care. The study objective was to systematically review evidence on the relationship between health insurance literacy and health care utilization.

METHODS: Medline, SCOPUS, Web of Science, CINAHL, PsychInfo, Cochrane Library, and reference lists of published literature were searched in August 2019. Quantitative, qualitative, and intervention studies that assessed the association of health insurance literacy as the exposure and health care utilization as the outcome were identified, without language or date restrictions. Outcomes were independently assessed by 2-3 reviewers.

RESULTS: Twenty-one studies including a total of 62,416 subjects met inclusion criteria: three interventional trials, two mixed-methods studies, and sixteen cross-sectional studies. Ten of thirteen preventive care studies suggested that higher health insurance literacy was associated with greater utilization of primary care and other preventive services. Eight of nine studies of care avoidance demonstrated that individuals with lower health insurance literacy were more likely to delay or avoid care. A few studies had mixed results regarding the utilization of emergency department, inpatient, and surgical care.

DISCUSSION: The emerging literature in this area suggests that health insurance literacy is an important factor that can enable effective utilization of health care, including primary care and preventive services. However, the literature is limited by a paucity of studies using validated tools that broadly measure health insurance literacy (rather than testing knowledge of specific covered services). Improving health insurance literacy of the general public and increasing plain language communication of health insurance plan features at the point of health care

Prior Presentation The findings in this review were previously presented at the American Public Health Association Annual Meeting (virtual), October 2020. Received November 8, 2020

Accepted April 8, 2021

Published online May 23, 2021 navigation may encourage more effective and costconscious utilization.

KEY WORDS: health insurance literacy; health care utilization; delay or avoidance of care; medication adherence.

J Gen Intern Med 37(2):375-89

DOI: $10.1007 / \mathrm{s} 11606-021-06819-0$

(C) This is a U.S. government work and not under copyright protection in the U.S.; foreign copyright protection may apply 2021

\section{INTRODUCTION}

In the wake of insurance coverage reforms and recent marked declines in employment and employer-sponsored insurance associated with the coronavirus disease 2019 (COVID-19) pandemic, US families are undergoing transitions in health insurance at a rapid pace. Such transitions may include losing health insurance coverage or switching plans from employersponsored insurance to Medicaid, the Affordable Care Act (ACA) Marketplace, or other types of coverage. The diversity of plans available to the American consumer underscores the importance of assessing individuals' understanding and navigation of plan features. Health insurance literacy (HIL) encompasses the knowledge of health insurance terms and the application of health insurance concepts. Numerous surveybased studies have found that HIL is low in the general population. ${ }^{1,2}$ Moreover, lower HIL is more prevalent amongst several populations with a high risk of unmet medical needs: those of lower socioeconomic status, ${ }^{3}$ racial/ethnic minorities, ${ }^{4}$ and older adults. ${ }^{5}$

Low HIL is problematic insofar as it could lead to ineffective and inefficient use of the health care system, resulting in financial and/or medical harm. Early work in the field of HIL has shown that individuals with lower HIL had greater difficulty choosing insurance plans than those with higher HIL, ${ }^{6}$ whereas those with higher HIL were found to be more capable of making choices on coverage provisions based on their personal values and medical history. ${ }^{7}$ Moreover, custom-made decision aids to guide plan selection that provided plan education, individualized cost estimates, and assessment of priorities led to increased 
HIL, decision self-efficacy, and confidence in plan selection when compared to unaided navigation of the health insurance Marketplace. ${ }^{8}$ However, understanding which insurance plan to purchase does not necessarily ensure effective utilization of the health care system. As one aspect of HIL is understanding how to apply insurance concepts, utilization is an important outcome measure to study.

Longstanding work in the related field of health literacy (which is distinct from HIL in its attention to knowledge about health, rather than health insurance) has demonstrated associations with health care utilization. A 2011 systematic review including 96 primary studies found that low health literacy was associated with suboptimal utilization: specifically, low health literacy was associated with greater emergency department utilization; more hospitalizations; lower utilization of preventative health care; and decreased adherence to prescription medications. ${ }^{9,10}$

HIL is a distinct form of literacy and self-efficacy from health literacy that may affect health care utilization in different and important ways. With its focus on patients' understanding of health insurance and health care, HIL encompasses functional knowledge of health plans' benefits, cost-sharing, and other details that could empower appropriate use of health care. Thus, studying the association between HIL and utilization can elucidate how (or whether) this functional knowledge impacts utilization. Given the increasing number of changes to health insurance and health care in the current era, the time is ripe to review the literature on HIL as an important factor in how, when, and why people utilize health care.

\section{METHODS}

We conducted a systematic review of the literature assessing the association between HIL and health care utilization. A search and extraction protocol was developed using guidelines from the Preferred Reporting Items for Systematic Reviews and Meta-Analyses (PRISMA) ${ }^{11}$ and made publicly available via PROSPERO prior to study initiation. ${ }^{12}$

\section{Literature Search}

The primary search was conducted in Medline (OVID). To develop the search strategy, the team examined relevant articles identified through team expertise and conducted exploratory searches on HIL and health care utilization in Medline. Since a MeSH term for "health insurance literacy" does not exist, the search combined literacy-related MeSH terms (e.g., health knowledge, attitudes, practice, health literacy) and keywords (e.g., confidence, attitude, understanding, ability, selfefficacy) with health insurance MeSH terms and keywords, to broadly capture the HIL literature. Additional database searches were translations of the core Medline search and included the following databases: Scopus (Elsevier), Web of Science (Clarivate), PsycINFO (EBSCOhost), CINAHL Complete (EBSCOhost), and Cochrane Central Register of
Controlled Trials (Wiley). Searches were run from database inception to the date of search: August 16, 2019. See Appendix 1 for further details of search terms. Reference lists of included documents were reviewed, and citation tracking of included documents took place in Scopus. Grey literature searching included conference proceedings. There were no restrictions on dates, language, country of origin, or patient population; intended subgroup analyses included the US vs. non-US and adult vs. pediatric populations.

\section{Study Selection}

The article titles and abstracts returned by the search were reviewed by three authors (BFY, JEL, MRF) in duplicate to determine if the studies appeared to contain any data on the association between HIL and health care utilization. Inclusion criteria were based on a broad definition of HIL: the knowledge and/or application of health insurance concepts, including laws, regulations, or policies governing health insurance. Any assessments of HIL were included, from validated measures such as the Health Insurance Literacy Measure $(\text { HILM })^{13}$ to author-created measures developed for specific studies. We also included measures of health literacy broadly so that full-text articles could be screened for potential secondary assessments of HIL.

Utilization was broadly defined as the use of and access to services for the purpose of preventing and curing health problems, promoting health and well-being, or obtaining information about one's health. ${ }^{14}$ All measures of health care utilization that met this definition were included (e.g., outpatient visits, emergency department visits, hospitalizations, avoidance of care, medication use/adherence, vaccinations). We did not include measures of health outcomes, health expenditures, quality of care, or medication management (as opposed to use). Disagreements regarding study inclusion or exclusion were resolved through discussion with a fourth reviewer (RT).

Full texts of the articles meeting the above selection criteria were uploaded into the DistillerSR ${ }^{15}$ online tool and reviewed by two authors (BFY, MRF) to determine if the studies met the inclusion criteria and reported on the appropriate exposure (HIL) and outcome (utilization). At this phase of review (see Fig. 1), qualitative studies (e.g., reports of focus groups) in which the relationship between HIL and utilization did not comprise a key theme or major finding were excluded. Studies in which HIL was treated as the outcome and not the exposure by the authors were excluded, as this was the reverse of the study objective. After full-text review, studies with measures of general health literacy (e.g., REALM, TOFHLA, NVS) but not including HIL were excluded. Studies with self-reported utilization were included, but studies that only assessed the intention or future plans to utilize health care were excluded. Disagreements regarding study inclusion at this phase of the review were resolved through discussion with a third or fourth reviewer (AMS, RT). 


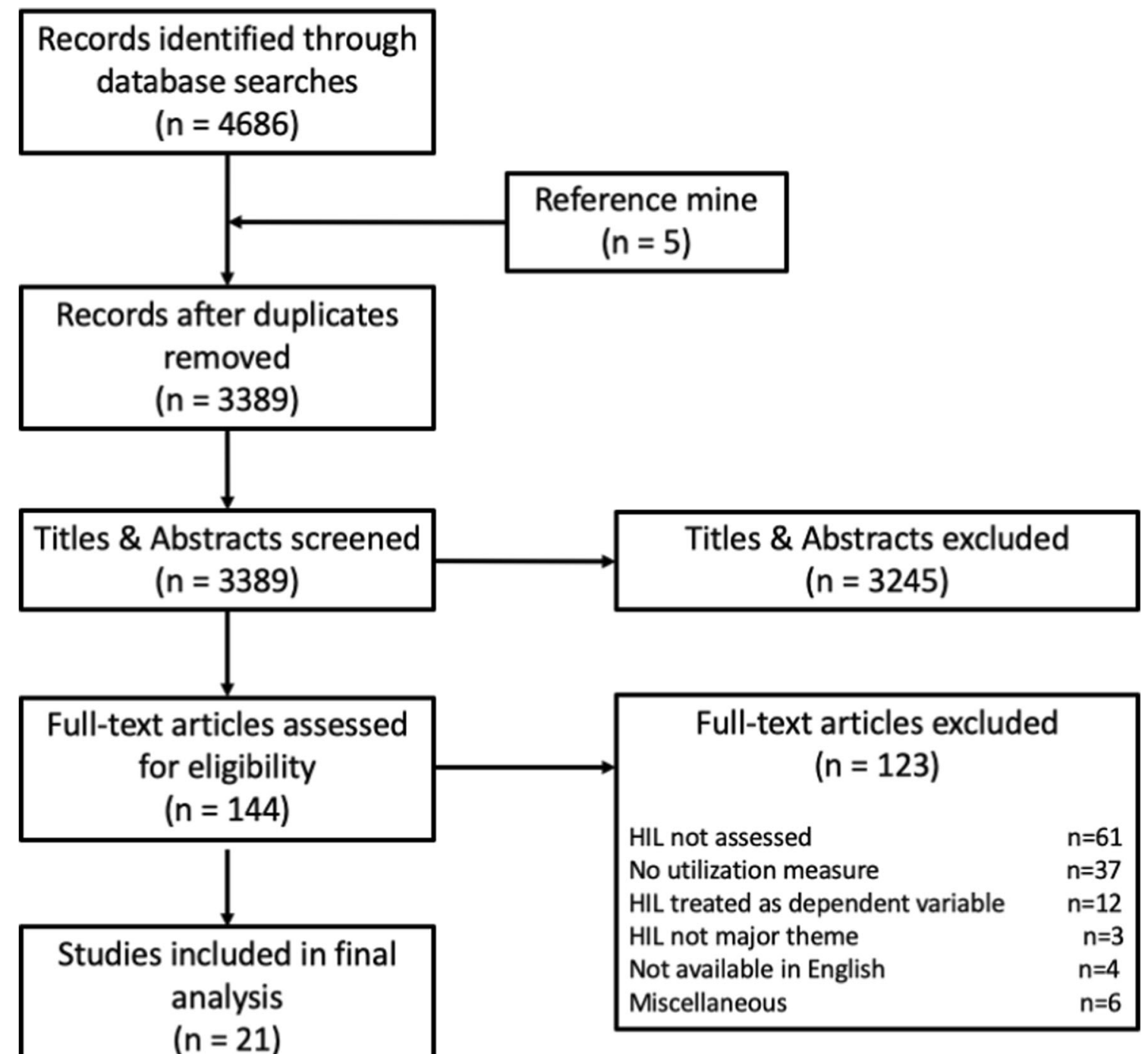

Figure 1 PRISMA diagram. Flow diagram of literature search, abstract screen, full article assessment for exclusion and inclusion criteria with most common reasons for exclusion detailed. Abbreviations: HIL, health insurance literacy.

\section{Data Extraction and Synthesis}

Data were extracted by two authors (BFY, MRF) using DistillerSR. Extracted variables included year, study type, data source, population (including sample size), country, how HIL was assessed, what type of utilization was assessed, how utilization was assessed, and the magnitude and significance of findings (Table 1). Studies were classified as a trial if there was an intervention with prospective randomization. HIL and health care utilization were considered significantly associated if $p<0.05$ or if the confidence interval of the comparison measure did not include the null value. Studies that did not find a statistically significant association were included. Risk of bias review and quality assessment was conducted using principles outlined in the Appraisal Tool for Cross-Sectional Studies (AXIS), finding that the included studies met between 75 and $95 \%$ of the twenty indicators of quality (Appendix 2). ${ }^{16}$

\section{RESULTS}

\section{Study Characteristics}

The initial search returned 3389 deduplicated results (Fig. 1). After reviewing the abstracts, 144 studies advanced to full-text review. Twenty-one studies ultimately met study criteria and were included in the final analysis. Of the twenty-one studies included in the final analysis (with publication dates from 2001 to 2019), three were prospective trials in which HIL was incorporated into an intervention, either as a direct objective of the study (e.g., sending participants reminders that a certain health care service is covered without any out-of-pocket costs) ${ }^{17,18}$ or as a consequence of the intervention (e.g., assigning case managers to high-risk patients to help them navigate their health insurance and health care). ${ }^{19}$ Table 1 provides a colorcoded summary of results, stratified by the utilization measures; Table 2 provides more granular details on each study, including year, population, and a detailed summary 
Table 1 Patterns of Association Between Health Insurance Literacy and Utilization

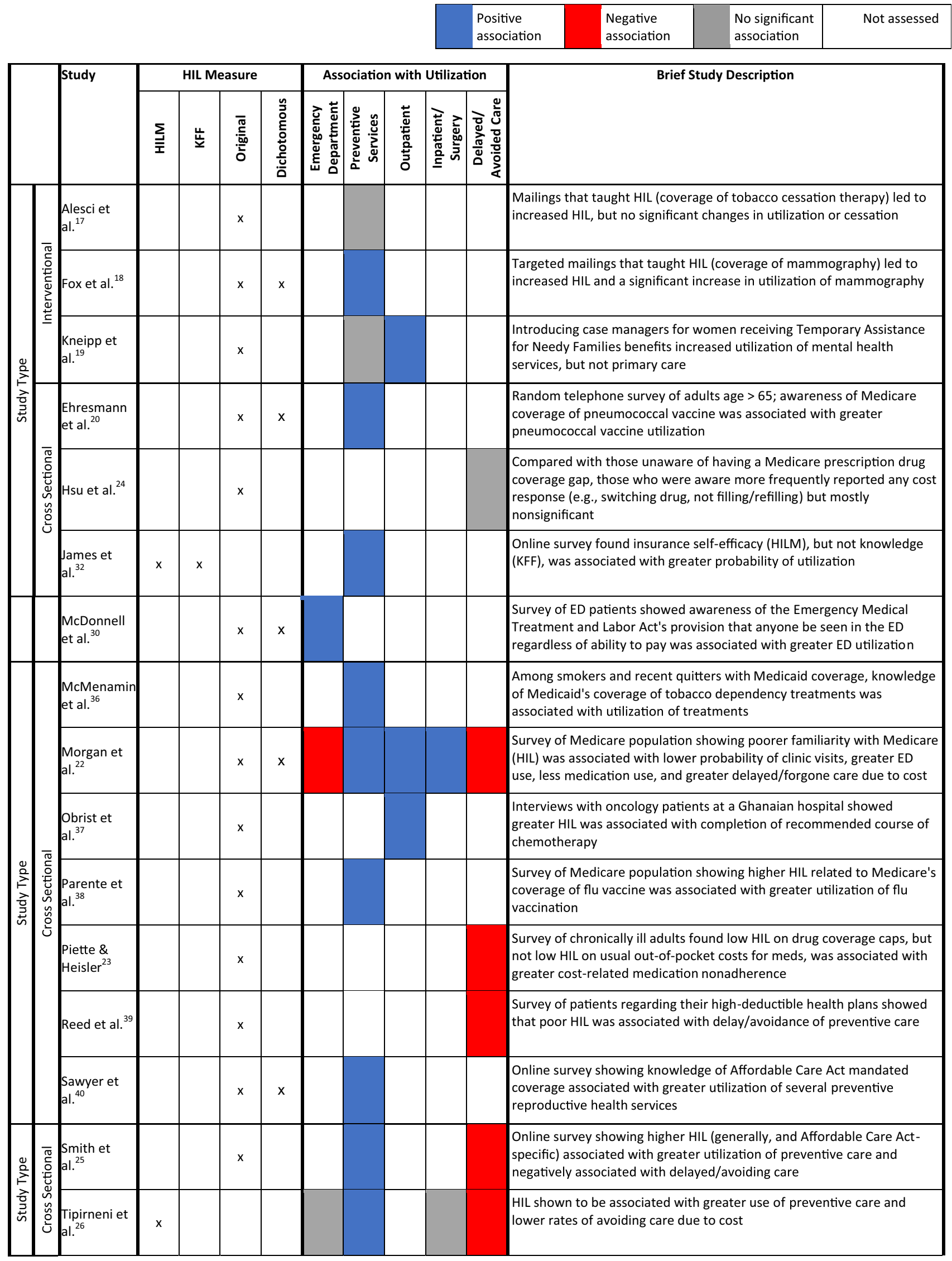


Table 1(continued)

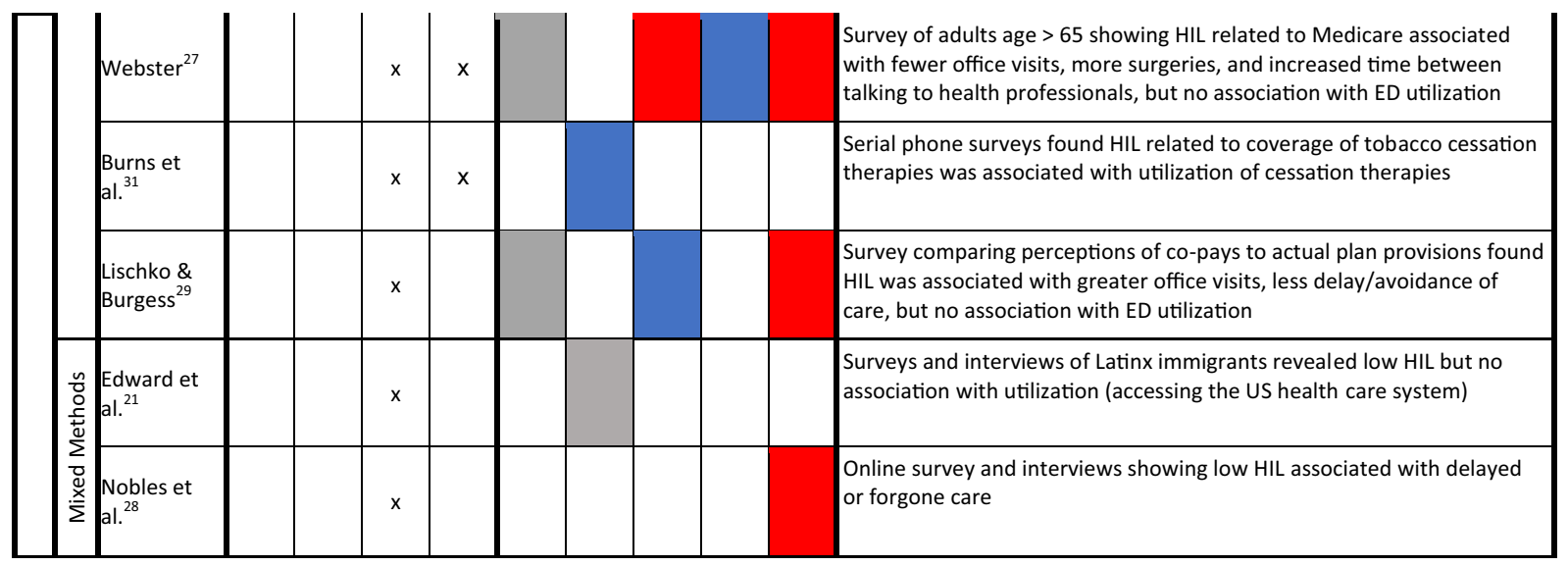

Abbreviations: HIL, health insurance literacy; ED, emergency department; HILM, Health Insurance Literacy Measure, a subjective measure of confidence in health insurance decision-making; KFF, Kaiser Family Foundation objective measure of health insurance knowledge

The 21 included studies are sorted by study type. The method of assessing HIL is denoted by Xs in the relevant cells. Dichotomous measures include assessments of HIL that asked a yes/no question about objective HIL knowledge or grouped respondents into high HIL/low HIL groups for analysis. Utilization measures were grouped into 5 categories: ED; preventive services (including primary care outpatient visits and use of specific services such as cancer screening, vaccinations, and tobacco cessation treatment); outpatient care (including subspecialty clinic visits and urgent visits); inpatient and surgical care, which were grouped together because of their higher costs and a paucity of studies; and delayed or avoidance of care

Legend: Blue = higher levels of HIL associated with increased utilization of the outcome measure; Red = higher levels of HIL associated with decreased utilization of the outcome measure (i.e., HIL is associated with fewer delays or avoidance of care, including medication use); Grey = no significant association between HIL and the outcome measure; White = association between HIL and the outcome measure was not assessed

of results. Two of the three interventional trials showed that increased HIL led to increased health care utilization. The remaining eighteen studies were cross-sectional analyses using surveys and/or semi-structured interviews. Most (18 of 21) of the included studies had multivariable adjustment incorporated into their analysis. Only one study was conducted outside of the USA (in Ghana), ${ }^{37}$ and only one study included a pediatric population, ${ }^{30}$ obviating the ability to perform subgroup analyses by country of origin or age of patient population. No purely qualitative studies met the final study inclusion criteria because their discussions of the relationship between HIL and utilization were ancillary, rather than constituting a major theme of the study. The most common reason for exclusion was that the study assessed health literacy, rather than HIL, or that it did not report utilization as an outcome (Fig. 1).

Overall, HIL was significantly associated with various types of health care utilization, with 19 of the 21 included studies showing statistically significant findings (Table 1 ). As Table 2 highlights, there was significant variability across studies in terms of the effect size of these relationships. Significant differences when comparing high HIL individuals to lower HIL individuals ranged from 1.5 to $36.1 \%$ absolute differences in health care utilization or medication use, 0.42 to 0.99 odds of delaying or foregoing medical care, and 1.08 to 11.86 greater odds of utilizing health services. A wide variety of utilization outcomes were measured in these studies, ranging from specific services (e.g., pneumococcal vaccination, ${ }^{20}$ mammography ${ }^{18}$ ) to broad measures (e.g., time since last utilization of health care $^{21}$ ).

\section{HIL and Preventative/Primary Care}

In general, higher levels of HIL were associated with greater utilization of outpatient and preventive care services. Of the 13 studies assessing the association between HIL and utilization of primary care or other preventive services, 10 showed a positive association between higher HIL and greater use of preventive services and 3 showed no significant difference.

\section{HIL and Avoidance of Needed Care}

Of the 9 studies assessing the association between HIL and delaying or avoiding care, 8 showed that low HIL was associated with delaying or avoiding care. ${ }^{21-28}$ For example, in a study of adults with high-deductible health plans in the Kaiser Permanente System, 24\% of those who mistakenly thought that their deductible applied to all office visits (when, in fact, preventive care visits had no out-of-pocket costs) said they delayed or avoided a preventive office visit because of cost, while only eight percent of those who correctly understood the cost-sharing scheme did so $(\mathrm{OR}=3.00) .{ }^{39}$ Two of these studies also demonstrated a significant association between low HIL and lower rates of medication adherence. ${ }^{22,23}$

\section{HIL and Acute Care Utilization}

Studies assessing the association between HIL and emergency department utilization $(n=5)$ had mixed findings, with three studies showing no significant association, ${ }^{26,27,29}$ one showing higher utilization, ${ }^{30}$ and one showing lower utilization. ${ }^{22}$ Of note, these five studies used five different assessments of HIL. One study, McDonnell et al., also assessed knowledge of 
Table 2 Detailed Description of Included Studies

\begin{tabular}{|c|c|c|c|c|c|c|c|c|}
\hline Study & Year & $\begin{array}{l}\text { Study type; } \\
\text { variable } \\
\text { adjustment } \\
\text { in analysis }\end{array}$ & $\begin{array}{l}\text { Population } \\
\text { (including } \mathbf{N} \text { ) }\end{array}$ & $\begin{array}{l}\text { HIL } \\
\text { assessment }\end{array}$ & $\begin{array}{l}\text { Type of } \\
\text { utilization } \\
\text { outcome }\end{array}$ & $\begin{array}{l}\text { Mode of } \\
\text { utilization } \\
\text { assessment }\end{array}$ & Study findings & $\begin{array}{l}\text { Magnitude of } \\
\text { association/ } \\
\text { effect }\end{array}$ \\
\hline Alesci et al. ${ }^{17}$ & 2004 & $\begin{array}{l}\text { Intervention; } \\
\text { multivariable } \\
\text { adjustment }\end{array}$ & $\begin{array}{l}\text { Smokers in } \\
\text { Minnesota } \\
\text { insurance plan } \\
(n=1930)\end{array}$ & $\begin{array}{l}\text { Self-reported } \\
\text { knowledge of } \\
\text { insurance } \\
\text { plan's } \\
\text { smoking } \\
\text { cessation } \\
\text { benefit and } \\
\text { 3-item } \\
\text { questionnaire } \\
\text { about benefit }\end{array}$ & $\begin{array}{l}\text { Tobacco } \\
\text { cessation } \\
\text { treatment; } \\
\text { tobacco } \\
\text { cessation }\end{array}$ & Survey & $\begin{array}{l}\text {-Knowledge of } \\
\text { plan benefit } \\
\text { greater in } \\
\text { control vs. } \\
\text { intervention } \\
\text { group with } \\
\text { targeted mailed } \\
\text { communication } \\
\text { but no } \\
\text { significant } \\
\text { differences in } \\
\text { tobacco benefit } \\
\text { utilization } \\
\text { between two } \\
\text { groups }\end{array}$ & $\begin{array}{l}\text { Bupropion } \\
\text { treatment in } \\
\text { past } 12 \\
\text { months } 23.1 \% \\
\text { in control vs. } \\
24.6 \% \text { in } \\
\text { intervention } \\
\text { group } \\
\text { ( } p=0.92) \text {; any } \\
\text { nicotine } \\
\text { replacement } \\
\text { treatment use } \\
\text { in past } 12 \\
\text { months } 25.9 \% \\
\text { vs. } 26.9 \% \text { in } \\
\text { control vs. } \\
\text { intervention } \\
\text { group } \\
(p=0.26)\end{array}$ \\
\hline Fox et al. $^{18}$ & 2001 & $\begin{array}{l}\text { Intervention; } \\
\text { multivariable } \\
\text { regression }\end{array}$ & $\begin{array}{l}\text { Women age > } \\
65 \text { in southern } \\
\text { California } \\
(n=917,922)\end{array}$ & $\begin{array}{l}\text { Knowledge } \\
\text { that screening } \\
\text { mammograms } \\
\text { are covered by } \\
\text { Medicare }\end{array}$ & $\begin{array}{l}\text { Mammography } \\
\text { in last } 2 \text { years }\end{array}$ & Survey & $\begin{array}{l}\text {-Mailing to } \\
\text { increase } \\
\text { knowledge that } \\
\text { Medicare pays } \\
\text { for breast } \\
\text { cancer } \\
\text { screening led to } \\
\text { increased } \\
\text { mammogram } \\
\text { use among } \\
\text { minorities who } \\
\text { received the } \\
\text { intervention } \\
\text { relative to } \\
\text { control group. } \\
\text {-However, the } \\
\text { intervention did } \\
\text { not increase } \\
\text { screening } \\
\text { amongst white } \\
\text { women }\end{array}$ & $\begin{array}{l}\text {-Black women } \\
\text { (OR 1.97) } \\
\text {-Hispanic } \\
\text { women (OR } \\
2.33 \text { ) } \\
\text {-White women } \\
\text { (OR 1.04) }\end{array}$ \\
\hline $\begin{array}{l}\text { Kneipp } \\
\text { et al. }\end{array}$ & 2011 & $\begin{array}{l}\text { Intervention; } \\
\text { multivariable } \\
\text { regression }\end{array}$ & $\begin{array}{l}\text { Women with } \\
\text { chronic health } \\
\text { conditions } \\
\text { receiving } \\
\text { TANF benefits } \\
\text { in Florida } \\
(\mathrm{n}=285)\end{array}$ & $\begin{array}{l}20 \text {-item } \\
\text { questionnaire } \\
\text { related to } \\
\text { Medicaid } \\
\text { coverage }\end{array}$ & $\begin{array}{l}\text { New mental } \\
\text { health visit, } \\
\text { primary care } \\
\text { routine, or } \\
\text { preventive visit }\end{array}$ & Survey & $\begin{array}{l}\text {-Subjects } \\
\text { receiving } \\
\text { intervention } \\
\text { with public } \\
\text { health nurse } \\
\text { case manager } \\
\text { who taught HIL } \\
\text { and other topics } \\
\text { were more } \\
\text { likely to have a } \\
\text { new mental } \\
\text { health visit but } \\
\text { not more likely } \\
\text { to have } \\
\text { preventive care } \\
\text { visit }\end{array}$ & $\begin{array}{l}\text {-New mental } \\
\text { health visit } \\
\text { (OR 1.92) } \\
\text {-Preventative } \\
\text { care visit (OR } \\
1.50)\end{array}$ \\
\hline $\begin{array}{l}\text { Ehresmann } \\
\text { et al. }\end{array}$ & 2001 & $\begin{array}{l}\text { Cross- } \\
\text { sectional, } \\
\text { quantitative; } \\
\text { multivariable } \\
\text { regression }\end{array}$ & $\begin{array}{l}\text { Adults age }> \\
65 \text { in } \\
\text { Minnesota } \\
(n=353)\end{array}$ & $\begin{array}{l}\text { Survey } \\
\text { assessing } \\
\text { awareness of } \\
\text { Medicare } \\
\text { coverage for } \\
\text { pneumococcal } \\
\text { vaccine }\end{array}$ & $\begin{array}{l}\text { Pneumococcal } \\
\text { vaccination }\end{array}$ & Survey & $\begin{array}{l}\text {-Awareness that } \\
\text { Medicare } \\
\text { covers } \\
\text { pneumococcal } \\
\text { vaccine } \\
\text { associated with } \\
\text { receipt of } \\
\text { pneumococcal } \\
\text { vaccine }\end{array}$ & OR 5.1 \\
\hline Hsu et al. ${ }^{24}$ & 2008 & $\begin{array}{l}\text { Cross- } \\
\text { sectional, } \\
\text { quantitative; } \\
\text { multivariable } \\
\text { regression }\end{array}$ & $\begin{array}{l}\text { Medicare } \\
\text { Advantage } \\
\text { beneficiaries } \\
\text { (age>65) in } \\
\text { Kaiser } \\
\text { Permanente }\end{array}$ & $\begin{array}{l}\text { After defining } \\
\text { coverage gap, } \\
\text { participants } \\
\text { were asked } \\
\text { whether their } \\
\text { drug plan }\end{array}$ & $\begin{array}{l}\text { Set of } \\
\text { medication } \\
\text { utilization } \\
\text { behaviors, } \\
\text { including } \\
\text { cost-coping }\end{array}$ & Interviews & $\begin{array}{l}\text {-Compared with } \\
\text { beneficiaries } \\
\text { unaware of } \\
\text { having a } \\
\text { Medicare } \\
\text { prescription }\end{array}$ & $\begin{array}{l}\text {-Any } \\
\text { behavioral } \\
\text { change: } \\
\text { difference of } \\
11.3 \%\end{array}$ \\
\hline
\end{tabular}


Table 2. (continued)

\begin{tabular}{|c|c|c|c|c|c|c|c|c|}
\hline Study & Year & $\begin{array}{l}\text { Study type; } \\
\text { variable } \\
\text { adjustment } \\
\text { in analysis }\end{array}$ & $\begin{array}{l}\text { Population } \\
\text { (including N) }\end{array}$ & $\begin{array}{l}\text { HIL } \\
\text { assessment }\end{array}$ & $\begin{array}{l}\text { Type of } \\
\text { utilization } \\
\text { outcome }\end{array}$ & $\begin{array}{l}\text { Mode of } \\
\text { utilization } \\
\text { assessment }\end{array}$ & Study findings & $\begin{array}{l}\text { Magnitude of } \\
\text { association/ } \\
\text { effect }\end{array}$ \\
\hline James et al. ${ }^{32}$ & 2018 & $\begin{array}{l}\text { Cross- } \\
\text { sectional, } \\
\text { quantitative; } \\
\text { multivariable } \\
\text { regression }\end{array}$ & $\begin{array}{l}\text { College } \\
\text { students at a } \\
\text { public } \\
\text { university in } \\
\text { Florida } \\
(\mathrm{n}=1450)\end{array}$ & $\begin{array}{l}\text { included such } \\
\text { a gap, at what } \\
\text { amount their } \\
\text { gap began and } \\
\text { ended, and } \\
\text { how much } \\
\text { they paid } \\
\text { before, during, } \\
\text { and after the } \\
\text { gap } \\
\text { KFF } \\
\text { knowledge } \\
\text { scale and } \\
\text { HILM }\end{array}$ & $\begin{array}{l}\text { behaviors (e.g., } \\
\text { switch to } \\
\text { cheaper med) } \\
\text { and decreased } \\
\text { adherence (e.g., } \\
\text { skip pills, didn't } \\
\text { fill) }\end{array}$ & Survey & $\begin{array}{l}\text { drug coverage } \\
\text { gap, those who } \\
\text { were aware } \\
\text { more frequently } \\
\text { reported any } \\
\text { behavior } \\
\text { change, } \\
\text { including } \\
\text { switching to a } \\
\text { cheaper drug } \\
\text {-No significant } \\
\text { association with } \\
\text { decreased med } \\
\text { adherence }\end{array}$ & $\begin{array}{l}\text {-Knowledge } \\
\text { (KFF) not } \\
\text { significantly } \\
\text { associated } \\
\text { with } \\
\text { utilization } \\
\text { including the } \\
\text { use of student } \\
\text { health services }\end{array}$ \\
\hline $\begin{array}{l}\text {-Higher } \\
\text { insurance } \\
\text { self-efficacy } \\
\text { (HILM) was } \\
\text { associated } \\
\text { with greater } \\
\text { probability of } \\
\text { overall } \\
\text { utilization } \\
\text { but not with } \\
\text { student } \\
\text { health } \\
\text { services }\end{array}$ & & & & & & & $\begin{array}{l}\text { HILM score } \\
\text { associated with } \\
\text { overall } \\
\text { utilization: OR } \\
1.91\end{array}$ & \\
\hline $\begin{array}{l}\text { McDonnell } \\
\text { et al. }\end{array}$ & 2013 & $\begin{array}{l}\text { Cross- } \\
\text { sectional, } \\
\text { quantitative; } \\
\text { multivariable } \\
\text { regression }\end{array}$ & $\begin{array}{l}\text { ED visitors (or } \\
\text { parents of } \\
\text { pediatric } \\
\text { patients) in } \\
\text { Utah }(n=4136)\end{array}$ & $\begin{array}{l}\text { Survey asking } \\
\text { if respondents } \\
\text { were aware of } \\
\text { a law that ED } \\
\text { must examine } \\
\text { and treat, } \\
\text { regardless of } \\
\text { insurance } \\
\text { status or ability } \\
\text { to pay }\end{array}$ & $\begin{array}{l}\text { ED visits in } \\
\text { prior year }\end{array}$ & Survey & $\begin{array}{l}\text {-Knowledge of } \\
\text { the Emergency } \\
\text { Medical } \\
\text { Treatment and } \\
\text { Labor Act } \\
\text { associated with } \\
\text { any ED } \\
\text { utilization } \\
\text {-Knowledge of } \\
\text { the Emergency } \\
\text { Medical } \\
\text { Treatment and } \\
\text { Labor Act also } \\
\text { associated with } \\
\text { high-frequency } \\
\text { ED utilization } \\
\text { of at least } 5 \\
\text { visits in last } \\
\text { year }\end{array}$ & $\begin{array}{l}\text {-Any ED } \\
\text { utilization: OR } \\
1.44 \\
\text {-High- } \\
\text { frequency ED } \\
\text { utilization: OR } \\
1.69\end{array}$ \\
\hline $\begin{array}{l}\text { McMenamin } \\
\text { et al. }\end{array}$ & 2006 & $\begin{array}{l}\text { Cross- } \\
\text { sectional, } \\
\text { quantitative; } \\
\text { multivariable } \\
\text { regression }\end{array}$ & $\begin{array}{l}\text { Current } \\
\text { smokers or } \\
\text { recent quitters, } \\
\text { ages } 18-64 \\
\text { with Medicaid } \\
\text { in the USA } \\
(n=820)\end{array}$ & $\begin{array}{l}\text { Questions } \\
\text { regarding } \\
\text { knowledge of } \\
\text { coverage for } \\
\text { several } \\
\text { tobacco } \\
\text { dependency } \\
\text { treatments } \\
\text { under their } \\
\text { state Medicaid } \\
\text { program }\end{array}$ & $\begin{array}{l}\text { Use of tobacco } \\
\text { dependency } \\
\text { treatment }\end{array}$ & Survey & $\begin{array}{l}\text {-Knowledge of } \\
\text { Medicaid } \\
\text { coverage } \\
\text { associated with } \\
\text { greater use of } \\
\text { tobacco } \\
\text { dependency } \\
\text { treatments, } \\
\text { including any } \\
\text { medication and } \\
\text { use of quitline }\end{array}$ & $\begin{array}{l}\text {-Use of } \\
\text { tobacco } \\
\text { dependency } \\
\text { treatments: } \\
\text { OR 3.0 } \\
\text {-Use of } \\
\text { quitline: OR } \\
3.5\end{array}$ \\
\hline $\begin{array}{l}\text { Morgan } \\
\text { et al.22 }\end{array}$ & 2008 & $\begin{array}{l}\text { Cross- } \\
\text { sectional, } \\
\text { quantitative; } \\
\text { multivariable } \\
\text { regression }\end{array}$ & $\begin{array}{l}\text { Medicare } \\
\text { beneficiaries in } \\
\text { the USA } \\
(n=2997)\end{array}$ & $\begin{array}{l}\text { Subjects asked } \\
\text { how familiar } \\
\text { they were with } \\
\text { Medicare and } \\
\text { Medicare } \\
\text { Advantage }\end{array}$ & $\begin{array}{l}\text { Clinic visits, } \\
\text { ED visits, } \\
\text { hospital } \\
\text { admissions in } \\
\text { the past year }\end{array}$ & Survey & $\begin{array}{l}\text { Lower } \\
\text { familiarity with } \\
\text { Medicare } \\
\text { associated with: } \\
\text {-Lower } \\
\text { likelihood of } \\
\text { clinic visits }\end{array}$ & $\begin{array}{l}\text {-Clinic visits: } \\
\text { OR } 0.67 \\
\text {-Prescription } \\
\text { drug use: OR } \\
0.58 \\
\text {-More } \\
\text { frequent ED } \\
\text { visits: } 2.88\end{array}$ \\
\hline
\end{tabular}


Table 2. (continued)

\begin{tabular}{|c|c|c|c|c|c|c|c|c|}
\hline Study & Year & $\begin{array}{l}\text { Study type; } \\
\text { variable } \\
\text { adjustment } \\
\text { in analysis }\end{array}$ & $\begin{array}{l}\text { Population } \\
\text { (including N) }\end{array}$ & $\begin{array}{l}\text { HIL } \\
\text { assessment }\end{array}$ & $\begin{array}{l}\text { Type of } \\
\text { utilization } \\
\text { outcome }\end{array}$ & $\begin{array}{l}\text { Mode of } \\
\text { utilization } \\
\text { assessment }\end{array}$ & Study findings & $\begin{array}{l}\text { Magnitude of } \\
\text { association// } \\
\text { effect }\end{array}$ \\
\hline & & & & & & & $\begin{array}{l}\text {-Lower } \\
\text { likelihood of } \\
\text { prescription } \\
\text { drug use } \\
\text {-Higher } \\
\text { likelihood of } \\
\text { more frequent } \\
\text { ED visits } \\
\text {-Non-significant } \\
\text { association with } \\
\text { greater inpatient } \\
\text { care } \\
\text {-Higher } \\
\text { likelihood of } \\
\text { delays due to } \\
\text { cost for clinic } \\
\text { visits, ED visits, } \\
\text { and inpatient } \\
\text { care }\end{array}$ & $\begin{array}{l}\text {-Delayed } \\
\text { clinic visits: } \\
\text { OR } 1.72 \\
\text {-Delayed ED } \\
\text { visits: OR } \\
2.07 \\
\text {-Delayed } \\
\text { inpatient care: } \\
\text { OR } 2.60\end{array}$ \\
\hline Obrist et al. ${ }^{37}$ & 2014 & $\begin{array}{l}\text { Cross- } \\
\text { sectional, } \\
\text { quantitative; } \\
\text { multivariable } \\
\text { regression }\end{array}$ & $\begin{array}{l}\text { Breast cancer } \\
\text { patients at } \\
\text { medical center } \\
\text { in Ghana } \\
(n=117)\end{array}$ & Interview & $\begin{array}{l}\text { Completion of } \\
\text { medically } \\
\text { recommended } \\
\text { breast cancer } \\
\text { treatment }\end{array}$ & $\begin{array}{l}\text { Medical } \\
\text { records }\end{array}$ & $\begin{array}{l}\text {-Patients who } \\
\text { completed } \\
\text { treatment were } \\
\text { significantly } \\
\text { more likely to } \\
\text { understand } \\
\text { what their } \\
\text { insurance } \\
\text { covered } \\
\text { regarding } \\
\text { surgery, } \\
\text { radiation, } \\
\text { chemotherapy, } \\
\text { and other } \\
\text { medications } \\
\text { than those who } \\
\text { did not } \\
\text { complete } \\
\text { treatment } \\
\text {-Those who } \\
\text { were unaware } \\
\text { of their } \\
\text { insurance } \\
\text { coverage policy } \\
\text { for breast care } \\
\text { had higher odds } \\
\text { of not } \\
\text { completing their } \\
\text { prescribed } \\
\text { breast cancer } \\
\text { treatment } \\
\text { protocol }\end{array}$ & $\begin{array}{l}-89.4 \% \text { of } \\
\text { patients who } \\
\text { completed } \\
\text { treatment } \\
\text { understood } \\
\text { coverage } \\
-67.74 \% \text { of } \\
\text { those who did } \\
\text { not complete } \\
\text { treatment } \\
\text { understood } \\
\text { coverage } \\
\text {-Awareness of } \\
\text { coverage } \\
\text { associated } \\
\text { with } \\
\text { completion of } \\
\text { treatment: OR } \\
11.859\end{array}$ \\
\hline $\begin{array}{l}\text { Parente } \\
\text { et al. }\end{array}$ & 2005 & $\begin{array}{l}\text { Cross- } \\
\text { sectional, } \\
\text { quantitative; } \\
\text { multivariable } \\
\text { regression }\end{array}$ & $\begin{array}{l}\text { Medicare } \\
\text { beneficiaries } \\
\text { (age> }>65) \text { in the } \\
\text { USA ( } n=7473 \text {; } \\
\text { with } n=4296 \\
\text { women for } \\
\text { mammogram } \\
\text { analysis) }\end{array}$ & $\begin{array}{l}\text { Medicare } \\
\text { beneficiary } \\
\text { survey with } \\
\text { test of } \\
\text { knowledge of } \\
\text { Medicare } \\
\text { coverage for } \\
\text { flu shot and } \\
\text { mammography }\end{array}$ & $\begin{array}{l}\text { Obtaining flu } \\
\text { shot, } \\
\text { mammogram }\end{array}$ & $\begin{array}{l}\text { Medicare } \\
\text { claims data }\end{array}$ & $\begin{array}{l}\text {-In both } \\
\text { analytic models, } \\
\text { individuals who } \\
\text { had knowledge } \\
\text { of the Medicare } \\
\text { flu shot benefit } \\
\text { had more flu } \\
\text { shots in the 12- } \\
\text { month period } \\
\text { studied }\end{array}$ & $\begin{array}{l}\text {-Model 1: } \\
0.092 \text { more flu } \\
\text { shots per year } \\
\text {-Model } 2 \text { : } \\
0.182 \text { more flu } \\
\text { shots per year }\end{array}$ \\
\hline $\begin{array}{l}\text { Piette and } \\
\text { Heisler }^{23}\end{array}$ & 2006 & $\begin{array}{l}\text { Cross- } \\
\text { sectional, } \\
\text { quantitative; } \\
\text { multivariable } \\
\text { regression }\end{array}$ & $\begin{array}{l}\text { Adults age }> \\
50 \text { in the USA } \\
\text { who had } \\
\text { prescription } \\
\text { drug coverage } \\
\text { and at least one } \\
\text { chronic } \\
\text { condition } \\
(n=3119)\end{array}$ & $\begin{array}{l}\text { Survey } \\
\text { questions } \\
\text { assessing } \\
\text { understanding } \\
\text { of usual cost } \\
\text { per } \\
\text { prescription } \\
\text { and knowledge } \\
\text { about drug }\end{array}$ & $\begin{array}{l}\text { Medication } \\
\text { adherence } \\
\text { (more } \\
\text { specifically, } \\
\text { cost-related) }\end{array}$ & Survey & $\begin{array}{l}\text {-Low HIL } \\
\text { regarding drug } \\
\text { coverage caps } \\
\text { associated with } \\
\text { cost-related } \\
\text { medication } \\
\text { nonadherence } \\
\text {-Low HIL } \\
\text { regarding usual } \\
\text { out-of-pocket }\end{array}$ & $\begin{array}{l}\text {-Low HIL } \\
\text { regarding drug } \\
\text { coverage gaps: } \\
\text { OR } 1.7 \\
\text {-Low HIL } \\
\text { regarding } \\
\text { usual out-of- } \\
\text { pocket costs: } \\
\text { OR } 1.0\end{array}$ \\
\hline
\end{tabular}


Table 2. (continued)

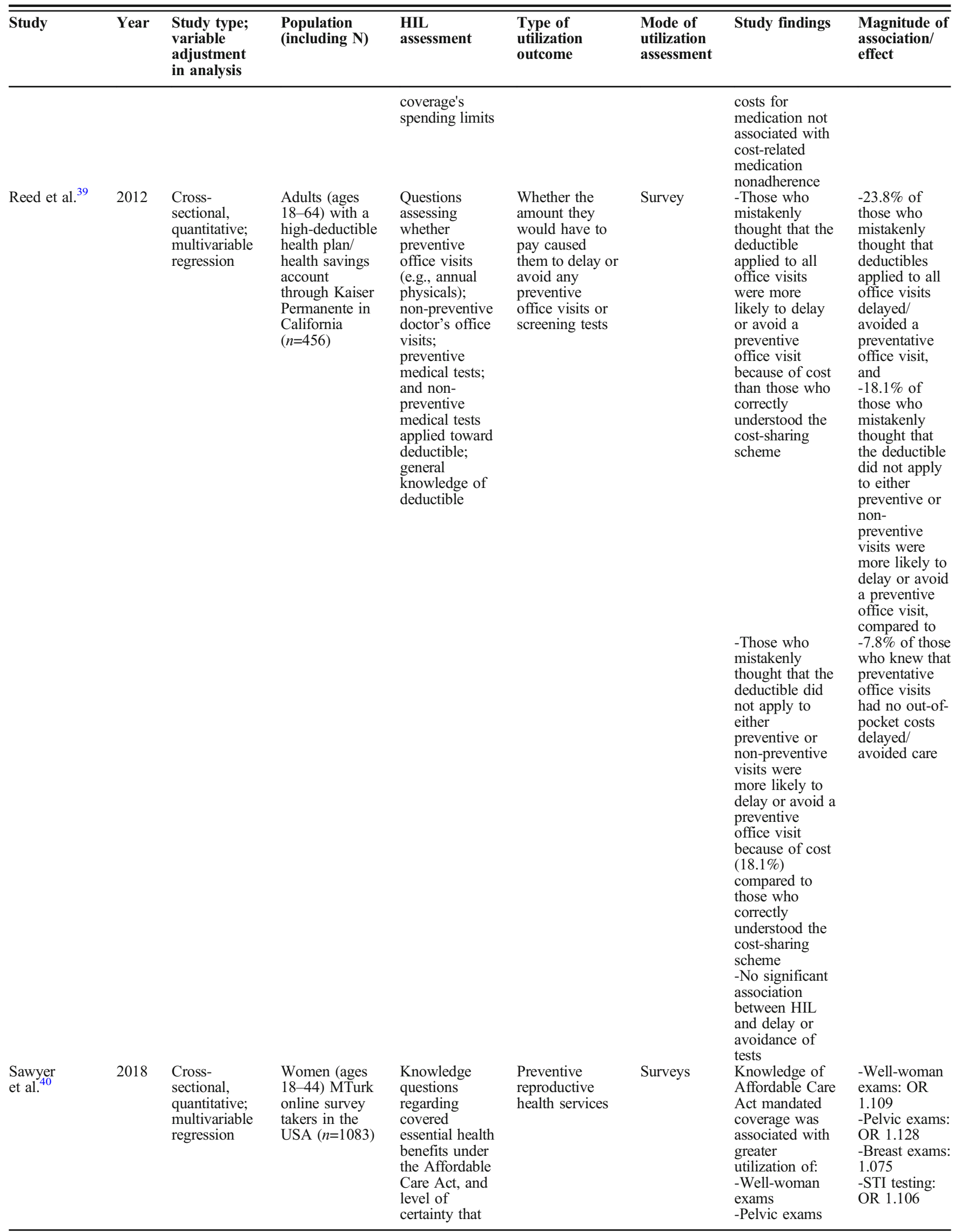


Table 2. (continued)

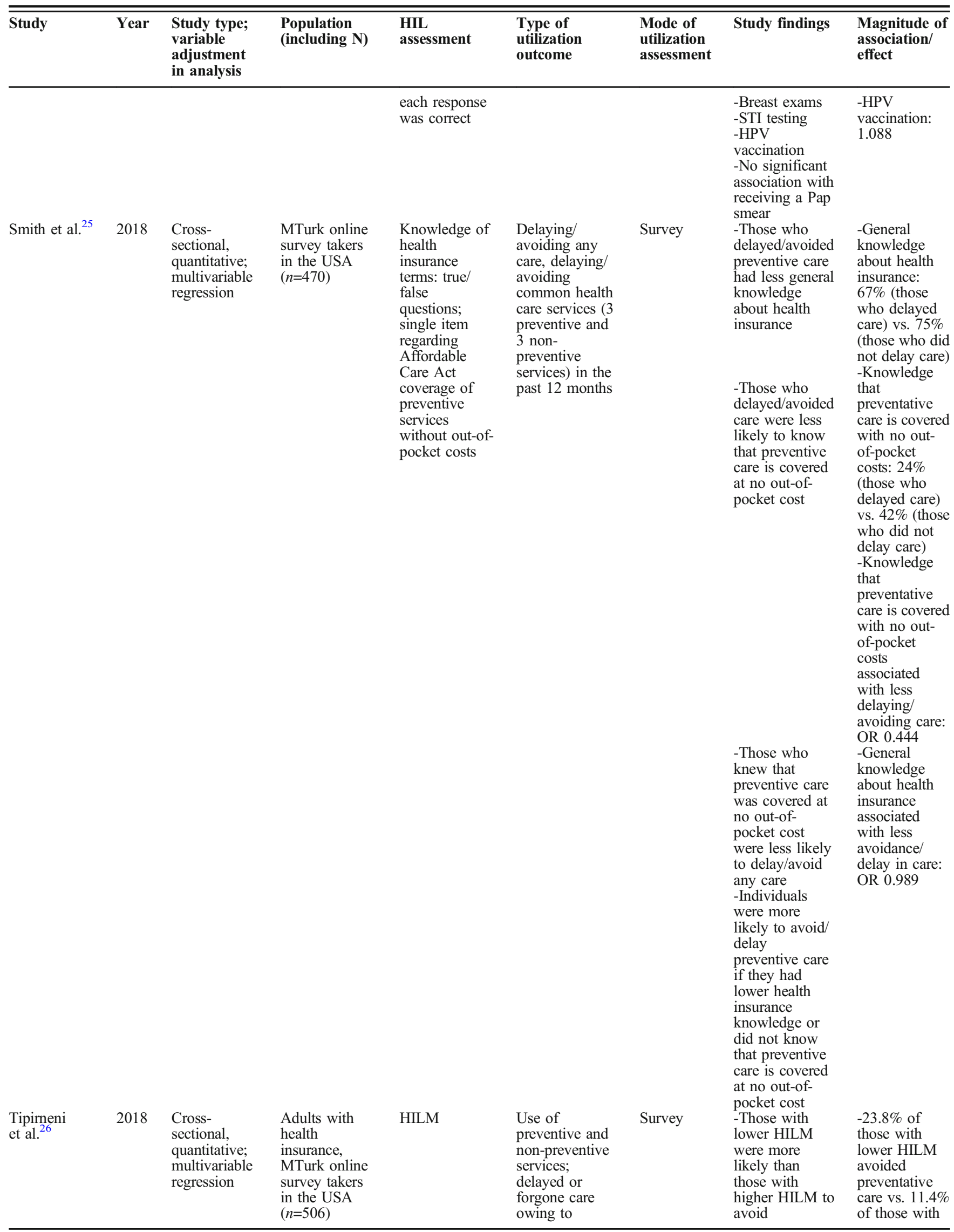


Table 2. (continued)

\begin{tabular}{|c|c|c|c|c|c|c|c|c|}
\hline Study & Year & $\begin{array}{l}\text { Study type; } \\
\text { variable } \\
\text { adjustment } \\
\text { in analysis }\end{array}$ & $\begin{array}{l}\text { Population } \\
\text { (including N) }\end{array}$ & $\begin{array}{l}\text { HIL } \\
\text { assessment }\end{array}$ & $\begin{array}{l}\text { Type of } \\
\text { utilization } \\
\text { outcome }\end{array}$ & $\begin{array}{l}\text { Mode of } \\
\text { utilization } \\
\text { assessment }\end{array}$ & Study findings & $\begin{array}{l}\text { Magnitude of } \\
\text { association/ } \\
\text { effect }\end{array}$ \\
\hline Webster $^{27}$ & 2011 & $\begin{array}{l}\text { Cross- } \\
\text { sectional, } \\
\text { quantitative; } \\
\text { multivariable } \\
\text { regression }\end{array}$ & $\begin{array}{l}\text { Adults age }>65 \\
\text { in the USA } \\
(N=30,002) \\
\text { from the } \\
\text { National } \\
\text { Health }\end{array}$ & $\begin{array}{l}\text { Knowledge } \\
\text { questions } \\
\text { related to type } \\
\text { of Medicare } \\
\text { coverage, } \\
\text { whether } \\
\text { enrolled in }\end{array}$ & $\begin{array}{l}\text { Number of } \\
\text { medical office } \\
\text { visits, ED visits, } \\
\text { time speaking } \\
\text { with health } \\
\text { professional, }\end{array}$ & Survey & $\begin{array}{l}\text {-Each 12-point } \\
\text { increase in } \\
\text { HILM score ( } 1 \\
\text { SD) was } \\
\text { associated with } \\
\text { lower likelihood } \\
\text { of delayed or } \\
\text { forgone care } \\
\text { owing to cost } \\
\text { for preventive } \\
\text { care } \\
\text {-Each 12-point } \\
\text { increase in } \\
\text { HILM score ( 1 } \\
\text { SD) was } \\
\text { associated with } \\
\text { lower likelihood } \\
\text { of delayed or } \\
\text { forgone care } \\
\text { owing to cost } \\
\text { for non- } \\
\text { preventive care } \\
\text {-HILM score } \\
\text { was associated } \\
\text { with a higher } \\
\text { likelihood of } \\
\text { preventive } \\
\text { services use, but } \\
\text { not with non- } \\
\text { preventive } \\
\text { services use } \\
\text {-Low HIL } \\
\text { associated with: } \\
\text { - Greater } \\
\text { number of } \\
\text { medical office } \\
\text { visits }\end{array}$ & $\begin{array}{l}\text { higher HILM } \\
\text { avoided } \\
\text { preventative } \\
\text { care } \\
-19.3 \% \text { of } \\
\text { those with } \\
\text { lower HILM } \\
\text { avoided non- } \\
\text { preventative } \\
\text { care vs. 12.6\% } \\
\text { of those with } \\
\text { higher HILM } \\
\text { avoided non- } \\
\text { preventative } \\
\text { care -Each SD } \\
\text { increase in } \\
\text { HILM } \\
\text { associated } \\
\text { with less } \\
\text { delayed/ } \\
\text { foregone } \\
\text { preventative } \\
\text { care due to } \\
\text { cost: OR 0.61 } \\
\text {-Each SD } \\
\text { increase in } \\
\text { HILM } \\
\text { associated } \\
\text { with less } \\
\text { delayed/ } \\
\text { foregone non- } \\
\text { preventative } \\
\text { care: OR } 0.71 \\
\text {-HILM score } \\
\text { associated } \\
\text { with utilizing } \\
\text { preventative } \\
\text { services: OR } \\
1.57\end{array}$ \\
\hline
\end{tabular}


Table 2. (continued)

\begin{tabular}{|c|c|c|c|c|c|c|c|c|}
\hline Study & Year & $\begin{array}{l}\text { Study type; } \\
\text { variable } \\
\text { adjustment } \\
\text { in analysis }\end{array}$ & $\begin{array}{l}\text { Population } \\
\text { (including N) }\end{array}$ & $\begin{array}{l}\text { HIL } \\
\text { assessment }\end{array}$ & $\begin{array}{l}\text { Type of } \\
\text { utilization } \\
\text { outcome }\end{array}$ & $\begin{array}{l}\text { Mode of } \\
\text { utilization } \\
\text { assessment }\end{array}$ & Study findings & $\begin{array}{l}\text { Magnitude of } \\
\text { association// } \\
\text { effect }\end{array}$ \\
\hline & & & $\begin{array}{l}\text { Interview } \\
\text { Survey }\end{array}$ & $\begin{array}{l}\text { Medicare } \\
\text { Advantage or } \\
\text { HMO, whether } \\
\text { referrals } \\
\text { needed for } \\
\text { specialty care, } \\
\text { and whether } \\
\text { paying for } \\
\text { supplemental } \\
\text { coverage }\end{array}$ & $\begin{array}{l}\text { and surgeries in } \\
\text { past } 12 \text { months }\end{array}$ & & $\begin{array}{l}\text {-More time } \\
\text { since last talked } \\
\text { with health } \\
\text { professional } \\
\\
\text {-Lower } \\
\text { likelihood of } \\
\text { talking with } \\
\text { health } \\
\text { professional } \\
\text {-Lower } \\
\text { likelihood of } \\
\text { surgery } \\
\text {-No } \\
\text { association with } \\
\text { ED visits in the } \\
\text { past twelve } \\
\text { months }\end{array}$ & $\begin{array}{l}1.3 \text { vs. } 1.2 \text { on } \\
\text { time scale } \\
\text { ranging from } 6 \\
\text { months or less } \\
\text { to never } \\
\text {-Likelihood of } \\
\text { talking with a } \\
\text { health } \\
\text { professional: } \\
44.1 \% \text { vs. } \\
47.1 \% \\
\text {-Likelihood of } \\
\text { surgery: } \\
18.0 \% \text { vs. } \\
20.5 \%\end{array}$ \\
\hline Burns et al. ${ }^{31}$ & 2005 & $\begin{array}{l}\text { Cross- } \\
\text { sectional, } \\
\text { quantitative; } \\
\text { adjusted for } \\
\text { survey } \\
\text { weights, no } \\
\text { covariates }\end{array}$ & $\begin{array}{l}\text { Wisconsin state } \\
\text { employees in } \\
\text { state-sponsored } \\
\text { health plan } \\
(2001 n=5609 \text {; } \\
2002 n=6518)\end{array}$ & $\begin{array}{l}\text { Yes no } \\
\text { question on } \\
\text { whether } \\
\text { insurance } \\
\text { covers list of } \\
\text { specific } \\
\text { tobacco } \\
\text { cessation } \\
\text { therapies }\end{array}$ & $\begin{array}{l}\text { Use of tobacco } \\
\text { cessation } \\
\text { medications }\end{array}$ & Survey & $\begin{array}{l}\text {-HIL related to } \\
\text { coverage of } \\
\text { tobacco } \\
\text { cessation } \\
\text { therapies } \\
\text { associated with } \\
\text { utilization of } \\
\text { tobacco } \\
\text { cessation } \\
\text { therapies }\end{array}$ & $\begin{array}{l}-39.6 \% \\
\text { utilization } \\
\text { among those } \\
\text { aware vs. } \\
3.5 \% \text { among } \\
\text { those unaware } \\
\text { of benefit }\end{array}$ \\
\hline $\begin{array}{l}\text { Lischko and } \\
\text { Burgess }^{29}\end{array}$ & 2010 & $\begin{array}{l}\text { Cross- } \\
\text { sectional, } \\
\text { quantitative; } \\
\text { multivariable } \\
\text { regression }\end{array}$ & $\begin{array}{l}\text { Massachusetts } \\
\text { state } \\
\text { employees (age } \\
<65) \\
\text { continuously } \\
\text { enrolled in } \\
\text { health plan for } \\
3 \text { years } \\
(n=1322)\end{array}$ & $\begin{array}{l}\text { Knowledge } \\
\text { questions } \\
\text { regarding co- } \\
\text { pays for } \\
\text { different } \\
\text { services }\end{array}$ & $\begin{array}{l}\text { ED or office } \\
\text { visits }\end{array}$ & $\begin{array}{l}\text { Claims } \\
\text { data, } \\
\text { Survey }\end{array}$ & $\begin{array}{l}\text {-Greater } \\
\text { knowledge of } \\
\text { costs was } \\
\text { associated with } \\
\text { utilization of } \\
\text { office visits } \\
\text {-Those who } \\
\text { overestimated } \\
\text { or accurately } \\
\text { knew co-pays } \\
\text { were more } \\
\text { likely to delay/ } \\
\text { avoid care than } \\
\text { those who } \\
\text { underestimated } \\
\text { co-pays } \\
\text {-Knowledge of } \\
\text { specific co-pays } \\
\text { had no } \\
\text { association with } \\
\text { office visits or } \\
\text { ED utilization }\end{array}$ & $\begin{array}{l}-0.0923 \text { more } \\
\text { office visits } \\
\text { for those with } \\
\text { the highest } \\
\text { level of cost- } \\
\text { sharing } \\
\text { knowledge vs. } \\
\text { no knowledge } \\
\text {-Those who } \\
\text { overestimated } \\
\text { (OR 2.47) or } \\
\text { accurately } \\
\text { knew co-pays } \\
\text { (OR 1.87) } \\
\text { were more } \\
\text { likely to delay/ } \\
\text { avoid office } \\
\text { visits }\end{array}$ \\
\hline $\begin{array}{l}\text { Edward } \\
\text { et al. }\end{array}$ & 2018 & $\begin{array}{l}\text { Cross- } \\
\text { sectional, } \\
\text { mixed } \\
\text { methods; } \\
\text { unadjusted }\end{array}$ & $\begin{array}{l}\text { Latinx } \\
\text { (primarily } \\
\text { Spanish } \\
\text { speaking) } \\
\text { adults } \\
\text { attending } \\
\text { health } \\
\text { insurance } \\
\text { enrollment } \\
\text { event }(n=139)\end{array}$ & $\begin{array}{l}\text { Subjects asked } \\
\text { to define } \\
\text { health } \\
\text { insurance } \\
\text { terms (copay, } \\
\text { premium, } \\
\text { deductible) }\end{array}$ & $\begin{array}{l}\text { Whether } \\
\text { participants had } \\
\text { accessed health } \\
\text { care in the USA }\end{array}$ & $\begin{array}{l}\text { Surveys, } \\
\text { semi- } \\
\text { structured } \\
\text { interviews }\end{array}$ & $\begin{array}{l}\text { No association } \\
\text { between HIL } \\
\text { and time since } \\
\text { last accessed } \\
\text { health care }\end{array}$ & $-\mathrm{N} / \mathrm{A}$ \\
\hline $\begin{array}{l}\text { Nobles } \\
\text { et al. }\end{array}$ & 2019 & $\begin{array}{l}\text { Cross- } \\
\text { sectional, } \\
\text { mixed } \\
\text { methods; } \\
\text { unadjusted }\end{array}$ & $\begin{array}{l}\text { Undergraduate } \\
\text { and graduate } \\
\text { students at a } \\
\text { single } \\
\text { university in }\end{array}$ & $\begin{array}{l}\text { Knowledge of } \\
\text { health } \\
\text { insurance } \\
\text { vocabulary } \\
\text { and ability to }\end{array}$ & $\begin{array}{l}\text { Delayed/ } \\
\text { forgone medical } \\
\text { care because of } \\
\text { confusion about }\end{array}$ & Survey & $\begin{array}{l}\text {-Low HIL } \\
\text { associated with } \\
\text { delayed or } \\
\text { forgone care }\end{array}$ & $\begin{array}{l}-24.4 \% \\
\text { indicated that } \\
\text { lack of } \\
\text { understanding } \\
\text { of their health }\end{array}$ \\
\hline
\end{tabular}


Table 2. (continued)

\begin{tabular}{|c|c|c|c|c|c|c|c|c|}
\hline Study & Year & $\begin{array}{l}\text { Study type; } \\
\text { variable } \\
\text { adjustment } \\
\text { in analysis }\end{array}$ & $\begin{array}{l}\text { Population } \\
\text { (including N) }\end{array}$ & $\begin{array}{l}\text { HIL } \\
\text { assessment }\end{array}$ & $\begin{array}{l}\text { Type of } \\
\text { utilization } \\
\text { outcome }\end{array}$ & $\begin{array}{l}\text { Mode of } \\
\text { utilization } \\
\text { assessment }\end{array}$ & Study findings & $\begin{array}{l}\text { Magnitude of } \\
\text { association// } \\
\text { effect }\end{array}$ \\
\hline & & & $\begin{array}{l}\text { Virginia } \\
(n=455)\end{array}$ & $\begin{array}{l}\text { apply } \\
\text { knowledge to } \\
\text { determine } \\
\text { cost-sharing, } \\
\text { self-rated } \\
\text { understanding } \\
\text { of insurance } \\
\text { terminology }\end{array}$ & $\begin{array}{l}\text { health insurance } \\
\text { plan }\end{array}$ & & & $\begin{array}{l}\text { insurance } \\
\text { stopped or } \\
\text { delayed them } \\
\text { from seeking } \\
\text { medical care } \\
\text { in the past }\end{array}$ \\
\hline
\end{tabular}

Abbreviations: HIL, health insurance literacy; OR, odds ratio; TANF, Temporary Assistance for Needy Families; HILM, Health Insurance Literacy Measure, a subjective measure of confidence in health insurance decision-making; KFF, Kaiser Family Foundation objective measure of health insurance knowledge; MTurk, Amazon Mechanical Turk; STI, sexually transmitted infection; HMO, health maintenance organization; ED, Emergency Department

Included studies are sorted by study type and presented in the same order as in Table 1

broader health insurance policies by measuring participants' awareness of the federal Emergency Medical Treatment and Labor Act (EMTALA), which requires emergency departments to care for patients regardless of insurance status or ability to pay; in this study, greater knowledge about the EMTALA policy was associated with higher levels of emergency department use. ${ }^{30}$ Two of three studies assessing utilization of inpatient or surgical care showed that HIL was associated with greater utilization, ${ }^{22,27}$ and one showed no statistical significance. ${ }^{26}$

\section{Measures of HIL Used Across Studies}

HIL was measured in a variety of ways in the included studies. We identified 21 different ways of measuring HIL across the 21 studies, 19 of which were novel measures created specifically for the study. To date, there is only one validated tool that measures HIL - the Health Insurance Literacy Measure-which assesses subjective confidence and behaviors associated with selecting and utilizing health insurance. ${ }^{13}$ Published in 2014, the Health Insurance Literacy Measure was used as a measurement tool in only two of the included studies. Most other studies used measures that were created by the authors for the specific purpose of the study. For example, Kneipp et al. ${ }^{19}$ created a 20 -item questionnaire about whether Medicaid covers specific services. Some studies assessed HIL very granularly; for example, Burns, Rosenberg, and Fiore were interested in how tobacco users utilized certain tobacco cessation therapies and assessed subjects' awareness of insurance coverage for those specific therapies. ${ }^{31}$

James et al. ${ }^{32}$ used two assessments of HIL, an objective measure developed by the Kaiser Family Foundation that assessed fact-based knowledge about insurance coverage (e.g., the definition of a deductible) ${ }^{2}$ and the Health Insurance Literacy Measure, a more subjective measure described above. The authors found that insurance knowledge (as measured by the Kaiser Family Foundation scale) was not associated with utilization patterns; however, insurance self-efficacy (as measured by the Health Insurance Literacy Measure) was positively associated with utilization. The only other study that utilized the Health Insurance Literacy Measure, Tipirneni et al., found that lower HIL was associated with greater likelihood of delayed or forgone care owing to cost and a lower likelihood of utilizing preventive, but not non-preventive, services. ${ }^{26}$

\section{DISCUSSION}

Our systematic review found that in most studies lower HIL was associated with lower health care utilization or greater avoidance of a wide variety of health care services. However, there is a dearth of literature in this area. Several studies demonstrated that low HIL was associated with underutilization of certain high-value services, including primary care visits, other preventive care visits, and adherence to prescription medication regimens for chronic conditions. Not surprisingly, greater specificity of the HIL measurement often had stronger associations with health care utilization patterns, though this was not consistent across studies. This highlights the need for HIL researchers to determine whether it is more useful to assess HIL in a context-specific way (e.g., knowledge of coverage of a specific service) or as a general skill or behavior that will be more likely to generalize across multiple health care contexts.

Another theme of our systematic review was that HIL may enable cost-conscious navigation of the health care system, and low HIL could be a barrier to effective care navigation. For example, eight of the nine studies that assessed delayed or forgone care found that lower HIL was associated with avoidance of needed care. This suggests that HIL is a key mediator of effective navigation of the many layers of the US health care system.

Our analysis of studies assessing HIL and health care utilization dovetails with other literature assessing the relationship between HIL and navigation of health insurance plan selection and affordability. Studies of factors influencing how people select insurance plans have shown that a lack of cost transparency $^{33}$ and a perceived lack of reliable information about how 
to distinguish different insurance plans ${ }^{34}$ are significant barriers towards selecting a plan. One study found that individuals over the age of 50 with concerns about the affordability of health insurance were more likely to delay or avoid care. ${ }^{35}$ Thus, improving HIL may enable individuals to exhibit costconscious care navigation on two fronts: choosing an appropriately tailored insurance plan and accessing and utilizing the health care system after obtaining insurance. Ideally, this would be facilitated by laws regulating how insurance plans communicate to consumers about the details of their benefits and cost-sharing. The ACA included provisions to improve the transparency of health plans' cost-sharing, but the implementation of these provisions has been thus far delayed. ${ }^{42}$

Our findings also beg the question: to improve the use of high-value care, should health care professionals aim to raise the level of HIL among all patients or focus on communicating health insurance terms and concepts in plain language? The intervention studies included in this review focused on the unique details of specific health insurance plans and specific covered services (e.g., education on Medicare's coverage of the influenza vaccine), which may not translate to broader use of high-value care (e.g., use of primary care vs. the emergency department). In addition, changes in the availability and type of insurance, medication formularies, in-network providers, and out-of-pocket costs of various services may make the implementation of any HIL intervention to encourage specific types of high-value care challenging and short-lived. Further studies could delineate whether HIL performs similarly across all populations and insurance models, or if specific populations may benefit from targeted interventions aimed at increasing specific aspects of HIL. ${ }^{42}$

As noted earlier, despite the importance of the topic, our review found that research about HIL is limited, both by the number of studies conducted to date and by the number of validated tools available to assess HIL. The substantial majority of studies in the systematic review relied upon a HIL assessment tool that was created by the authors specifically for that study, making comparisons across HIL studies challenging. Several of the reported measures were narrow both in their assessment of HIL (e.g., knowledge of out-of-pocket costs for a specific therapy) and in their examination of health care utilization (e.g., utilization of that specific therapy). A recent study that was published after the date of our search compared two other HIL scales (likelihood of utilization vs. confidence in utilization) and found divergent results in individuals' delaying care or reporting burdensome medical bills. ${ }^{41}$ This study highlights the difficulty of developing an assessment tool that captures both the broad concept of HIL and the specific applicability to health insurance plan selection and health care navigation. Even the validated Health Insurance Literacy Measure tool focuses on measuring confidence in HIL (a subjective measure) but lacks a knowledgebased/objective assessment. Development and validation of new comprehensive measures of HIL are needed to advance this emerging and valuable field. Despite these differences in measurement, 19 of the 21 studies reported a statistically significant outcome, although there may be a publication bias that has led other studies with negative or null results to remain unpublished.

Our systematic review was conducted with a pre-specified protocol, comprehensive review of the literature, and coding my multiple reviewers. However, our review was primarily focused on biomedical and health care databases; we did not conduct a search with other types of databases such as those used in legal literature.

\section{CONCLUSION}

In summary, the literature addressing the association between HIL and health care utilization is limited and lacks standardized measures to assess HIL. This review of the current literature suggests that low HIL is a barrier to effective utilization of important health care services such as primary care, preventive services, medication adherence, and minimizing delays or avoidance of care for urgent needs. Thus, improving HIL and increasing plain language communication of health insurance plan features at the point of care navigation may be effective strategies to encourage more effective and costconscious utilization.

Author Contribution: All those who contributed to this manuscript are listed as authors.

Corresponding Author: Renuka Tipirneni, MD, MSc; Institute for Healthcare Policy and Innovation, University of Michigan, Ann Arbor, MI, USA (e-mail: rtipirne@med.umich.edu).

Supplementary InformationThe online version contains supplementary material available at https://doi.org/10.1007/s11606-02106819-0.

Funding The study was supported by the Department of Internal Medicine, University of Michigan. Dr. Tipirneni is additionally supported by a K08 Clinical Scientist Development Award from the National Institute on Aging. Dr. Scherer is additionally supported by a KO1 Mentored Research Scientist Award from the National Institute on Aging and a UO1 Cooperative Agreement with the Centers for Disease Control and Prevention.

\section{Declarations:}

Conflict of Interest: The authors declare that they do not have a conflict of interest.

\section{REFERENCES}

1. Loewenstein G, Friedman JY, McGill B, et al. Consumers' misunderstanding of health insurance. J Health Econ 2013;32(5):850-62.

2. Norton M, Hamel L, Brodie M. Assessing Americans' familiarity with health insurance terms and concepts. Kaiser Family Foundation. 2014. https://www.kff.org/health-reform/poll-finding/assessing-americansfamiliarity-with-health-insurance-terms-and-concepts. Accessed August 24, 2020. 
3. Feinberg I, Greenberg D, Tighe EL, Ogrodnick MM. Health Insurance Literacy and Low Wage Earners: Why Reading Matters. Adult Literacy Education 2019;1(2):4-18.

4. Williams CB, Pensa MA, Olson DP. Health insurance literacy in community health center staff. J Public Health 2020: 1-5.

5. McCormack L, Bann C, Uhrig J, Berkman N, Rudd R. Health insurance literacy of older adults. J Consum Aff 2009;43(2):223-48.

6. Ubel PA, Comerford DA, Johnson E. Healthcare.gov 3.0-Behavioral economics and insurance exchanges. N Engl J Med 2015; 372:695-8.

7. Politi MC, Kaphingst KA, Liu JE, et al. A randomized trial examining three strategies for supporting health insurance decisions among the uninsured. Med Decis Mak 2016;36(7):911-22.

8. Politi MC, Kuzemchak MD, Liu J, et al. Show Me My Health Plans: Using a Decision Aid to Improve Decisions in the Federal Health Insurance Marketplace. MDM Policy Pract. 2016;1(1):2381468316679998.

9. Berkman ND, Sheridan SL, Donahue KE, Halpern DJ, Crotty K. Low health literacy and health outcomes: an updated systematic review. Ann Intern Med 2011;155(2):97-107.

10. Walters R, Leslie SJ, Polson R, Cusack T, Gorely T. Establishing the efficacy of interventions to improve health literacy and health behaviours: a systematic review. BMC Public Health 2020;20(1):1040.

11. PRISMA checklist. http://prisma-statement.org/. Accessed August 6, 2020.

12. Luster J, Smith J, Yagi B, Farron M, Scherer A, Tipirneni R. Health insurance literacy and health care utilization: a systematic review. PROSPERO 2020 CRD42020147793 Available from: https://www.crd. york.ac.uk/prospero/display_record.php?ID=CRD42020147793.

13. Paez KA, Mallery CJ, Noel $\mathbf{H}$, et al. Development of the Health Insurance Literacy Measure (HILM): conceptualizing and measuring consumer ability to choose and use private health insurance. $J$ Health Commun. 2014;19 Suppl 2(sup2):225-39.

14. Carrasquillo 0 . Health Care Utilization. In: Gellman MD, Turner JR, eds. Encyclopedia of Behavioral Medicine. New York: Springer New York; 2013:909-10

15. Distiller-Systematic Review. Evidence Partners. https://www. evidencepartners.com/products/distillersr-systematic-review-software/. Accessed August 24, 2020.

16. Downes MJ, Brennan ML, Williams HC, Dean RS. Development of a critical appraisal tool to assess the quality of cross-sectional studies (AXIS). BMJ Open 2016;6(12):e011458.

17. Alesci NL, Boyle RG, Davidson G, Solberg LI, Magnan S. Does a health plan effort to increase smokers' awareness of cessation medication coverage increase utilization and cessation? Am J Health Promot 2004; 18(5):366-9.

18. Fox SA, Stein JA, Sockloskie RJ, Ory MG. Targeted mailed materials and the Medicare beneficiary: increasing mammogram screening among the elderly. Am J Public Health 2001;91(1):55-61.

19. Kneipp SM, Kairalla JA, Lutz BJ, et al. Public health nursing case management for women receiving temporary assistance for needy families: a randomized controlled trial using community-based participatory research. Am J Public Health 2011;101(9): 1759-68.

20. Ehresmann KR, Ramesh A, Como-Sabetti K, Peterson DC, Whitney CG, Moore KA. Factors associated with self-reported pneumococcal immunization among adults 65 years of age or older in the Minneapolis-St. Paul metropolitan area. Prev Med 2001;32(5):409-15.

21. Edward J, Morris S, Mataoui F, Granberry P, Williams MV, Torres I. The impact of health and health insurance literacy on access to care for Hispanic/Latino communities. Public Health Nurs 2018;35(3):176-83.

22. Morgan RO, Teal CR, Hasche JC, et al. Does poorer familiarity with Medicare translate into worse access to health care? J Am Geriatr Soc 2008;56(11):2053-60.

23. Piette JD, Heisler M. The relationship between older adults' knowledge of their drug coverage and medication cost problems. J Am Geriatr Soc 2006;54(1):91-6.
24. Hsu J, Fung V, Price M, et al. Medicare beneficiaries' knowledge of Part D prescription drug program benefits and responses to drug costs. JAMA. 2008;299(16):1929-36.

25. Smith KT, Monti D, Mir N, Peters E, Tipirneni R, Politi MC. Access is necessary but not sufficient: factors influencing delay and avoidance of health care services. MDM Policy Pract 2018;3(1):2381468318760298.

26. Tipirneni R, Politi MC, Kullgren JT, Kieffer EC, Goold SD, Scherer AM. Association between health insurance literacy and avoidance of health care services owing to cost. JAMA Netw Open 2018;1(7):e184796.

27. Webster NJ. Medicare Knowledge and Health Service Utilization Among Older Adults. In: Jacobs Kronenfeld J, ed. Access To Care and Factors That Impact Access, Patients as Partners In Care and Changing Roles of Health Providers. Emerald Group Publishing Limited; 2011:273-97.

28. Nobles AL, Curtis BA, Ngo DA, Vardell E, Holstege CP. Health insurance literacy: a mixed methods study of college students. J Am Coll Heal 2019;67(5):469-78.

29. Lischko AM, Burgess Jr JF. Knowledge of cost sharing and decisions to seek care. Am J Manag Care 2010;16(4):298-304.

30. McDonnell WM, Gee CA, Mecham N, Dahl-Olsen J, Guenther E. Does the Emergency Medical Treatment and Labor Act affect emergency department use? J Emerg Med 2013;44(1):209-16.

31. Burns ME, Rosenberg MA, Fiore MC. Use of a new comprehensive insurance benefit for smoking-cessation treatment. Prev Chronic Dis. 2005;2(4). https://www.cdc.gov/pcd/issues/2005/oct/05_0007.htm

32. James TG, Sullivan MK, Dumeny L, Lindsey $\mathbf{K}$, Cheong J, Nicolette G. Health insurance literacy and health service utilization among college students. J Am Coll Heal 2020;68(2):200-6.

33. George N, Grant R, James A, Mir N, Politi MC. Burden associated with selecting and using health insurance to manage care costs: results of a qualitative study of nonelderly cancer survivors. Med Care Res Rev. 2018;1077558718820232.

34. Furtado KS, Kaphingst KA, Perkins H, Politi MC. Health insurance information-seeking behaviors among the uninsured. J Health Commun 2016;21(2):148-58.

35. Tipirneni R, Solway E, Malani P, et al. Health insurance affordability concerns and health care avoidance among US adults approaching retirement. JAMA Netw Open 2020;3(2):e1920647.

36. McMenamin SB, Halpin HA, Bellows NM. Knowledge of Medicaid coverage and effectiveness of smoking treatments. Am J Prev Med. 2006;31(5):369-74.

37. Obrist M, Osei-Bonsu E, Ahwah B, et al. Factors related to incomplete treatment of breast cancer in Kumasi, Ghana Breast 2014;23(6):821-8.

38. Parente ST, Salkever DS, DaVanzo J. The role of consumer knowledge of insurance benefits in the demand for preventive health care among the elderly. Health Econ 2005; 14(1):25-38.

39. Reed ME, Graetz I, Fung V, Newhouse JP, Hsu J. In consumer-directed health plans, a majority of patients were unaware of free or low-cost preventive care. Health Aff. 2012;31(12):2641-8.

40. Sawyer AN, Kwitowski MA, Benotsch EG. Are you covered? associations between patient protection and affordable care act knowledge and preventive reproductive service use. Am J Health Promot 2018;32(4):90615.

41. Call KT, Conmy A, Alarcón G, Hagge SL, Simon AB. Health insurance literacy: How best to measure and does it matter to health care access and affordability? Res Social Adm Pharm. 2020.

42. Jenny HE, Jenny BE. A Healthy Dose of Price Transparency in US Health Care Services. JAMA Surg 2020;155(7):544-545.

Publisher's Note: Springer Nature remains neutral with regard to jurisdictional claims in published maps and institutional affiliations. 